\title{
Modeling Human Sentence Processing with Left-Corner Recurrent Neural Network Grammars
}

\author{
Ryo Yoshida ${ }^{1}$ and Hiroshi Noji ${ }^{2}$ and Yohei Oseki ${ }^{1}$ \\ ${ }^{1}$ The Univertisy of Tokyo \\ ${ }^{2}$ Artificial Intelligence Research Center, AIST \\ \{yoshiryo0617, oseki\}@g.ecc.u-tokyo.ac.jp \\ hiroshi.noji@aist.go.jp
}

\begin{abstract}
In computational linguistics, it has been shown that hierarchical structures make language models (LMs) more human-like. However, the previous literature has been agnostic about a parsing strategy of the hierarchical models. In this paper, we investigated whether hierarchical structures make LMs more human-like, and if so, which parsing strategy is most cognitively plausible. In order to address this question, we evaluated three LMs against human reading times in Japanese with head-final leftbranching structures: Long Short-Term Memory (LSTM) as a sequential model and Recurrent Neural Network Grammars (RNNGs) with top-down and left-corner parsing strategies as hierarchical models. Our computational modeling demonstrated that left-corner RNNGs outperformed top-down RNNGs and LSTM, suggesting that hierarchical and leftcorner architectures are more cognitively plausible than top-down or sequential architectures. In addition, the relationships between the cognitive plausibility and (i) perplexity, (ii) parsing, and (iii) beam size will also be discussed.
\end{abstract}

\section{Introduction}

It has been debated in computational linguistics whether language models (LMs) become more human-like by explicitly modeling hierarchical structures of natural languages. Previous work has revealed that while sequential models such as recurrent neural networks (RNNs) can successfully predict human reading times (Frank and Bod, 2011), there is an advantage in explicitly modeling hierarchical structures (Fossum and Levy, 2012). More recently, RNNs that explicitly model hierarchical structures, namely Recurrent Neural Network Grammars (RNNGs, Dyer et al., 2016), have attracted considerable attention, effectively capturing grammatical dependencies (e.g., subject-verb agreement) much better than RNNs in targeted syntactic evaluations (Kuncoro et al., 2018; Wilcox et al., 2019). In addition, Hale et al. (2018) showed that RNNGs can successfully predict human brain activities, and recommended RNNGs as "a mechanistic model of the syntactic processing that occurs during normal human sentence processing."

However, this debate has focused almost exclusively on the dichotomy between the hierarchical and sequential models, without reference to the parsing strategies among the hierarchical models. Specifically, although Dyer et al. (2016) and Hale et al. (2018) adopted the vanilla RNNG with a top-down parsing strategy for English with headinitial right-branching structures, Abney and Johnson (1991) and Resnik (1992) suggested that the top-down parsing strategy is not optimal for headfinal left-branching structures, and alternatively proposed the left-corner parsing strategy as more human-like parsing strategy.

In this paper, we investigate whether hierarchical structures make LMs more human-like, and if so, which parsing strategy is most cognitively plausible. In order to address this question, we evaluate three LMs against human reading times in Japanese with head-final left-branching structures: Long Short-Term Memory (LSTM) as a sequential model and Recurrent Neural Network Grammars (RNNGs) with top-down and left-corner parsing strategies as hierarchical models.

\section{Linking hypothesis}

It is well established in psycholinguistics that humans predict next segments during sentence processing, and the less predictable the segment is, the more surprising that segment is. Surprisal theory (Hale, 2001; Levy, 2008) quantifies this predictability of the segment as $-\log p$ (segment|context), an information-theoretic complexity metric known as surprisal. In line with the previous literature (e.g., Smith and Levy, 2013), we employed this metric to logarithmically link probabilities estimated from LMs with cognitive efforts measured 
from humans. Intuitively, the cognitively plausible LMs will compute surprisals with similar trends as human cognitive efforts. Computational models of human sentence processing have been explored by comparing surprisals from various LMs with reading times (e.g., Frank and Bod, 2011) and brain activities (e.g., Frank et al., 2015).

\section{Methods}

\subsection{Language models}

Long Short-Term Memory (LSTM): LSTMs are a sequential model that does not model hierarchical structures. We used a 2-layer LSTM with 256 hidden and input dimensions. The implementation by Gulordava et al. (2018) was employed.

Recurrent Neural Network Grammar (RNNG): RNNGs are a hierarchical model that explicitly models hierarchical structures. We experimented with two types of stack-only RNNGs (Kuncoro et al., 2017): top-down RNNGs and left-corner RNNGs (Kuncoro et al., 2018). ${ }^{1}$ We used RNNGs that had a 2-layer stack LSTM with 256 hidden and input dimensions. The implementation by Noji and Oseki (2021) was employed. Word-synchronous beam search (Stern et al., 2017) was used for inference. RNNGs were evaluated with six beam sizes $k=\{100,200,400,600,800,1000\} .^{2}$

\subsection{Data sets}

Training data: All LMs were trained on the National Institute for Japanese Language and Linguistics Parsed Corpus of Modern Japanese (NPCMJ), that comprises 67,018 sentences annotated with tree structures. ${ }^{3}$ The sentences were split into subwords by a byte-pair encoding (Sennrich et al., 2016). LSTM used only terminal subwords, while RNNGs used terminal subwords and tree structures, both of which were trained sentence-level for 40 epochs and 3 times with different random seeds. ${ }^{4}$

\footnotetext{
${ }^{1}$ Resnik (1992) suggested that an arc-eager left-corner parsing strategy is cognitively plausible. Jin and Schuler (2020) implemented an incremental neural parser that builds tree structures with the arc-eager left-corner parsing strategy, but it requires an extremely large beam size to achieve the reasonable parsing accuracy. Thus, in this paper, we employed arc-standard left-corner RNNGs as an approximation to the arc-eager left-corner parsing strategy that delayed attachments (Kuncoro et al., 2018).

${ }^{2} k$ means the action beam size. We set the word beam size to $k / 10$ and the fast-track to $k / 100$ (Stern et al., 2017).

${ }^{3}$ http: / / npcmj.ninjal.ac.jp

${ }^{4}$ Following Frank and Bod (2011), traces and semantic information were removed in the way described in Manning and Schutze (1999).
}

Reading time data: All LMs were evaluated against first pass reading times from BCCWJEyeTrack (Asahara et al., 2016), that comprises 218 sentences annotated with eye-tracking reading times at each phrasal unit. Following Asahara et al. (2016), the data points (i) not corresponding to the main text or (ii) not fixated were removed. In addition, following Fossum and Levy (2012), the data points that contained subwords "unknown" to the LMs were also removed. Consequently, we included 12,114 data points in the statistical analyses among 19,717 data points in total.

\subsection{Evaluation metrics}

Psychometric predictive power: We evaluated how well surprisal ( $-\log p$ (segment|context)) from each LM could predict human reading times. LMs process the sentences subword-by-subword, while reading times are annotated phrase-by-phrase. Thus, following Wilcox et al. (2020), the phrasal surprisal $I(p)$ was computed as the cumulative sum of surprisals of its constituent subwords $w_{l}, w_{l+1}, \cdots, w_{m}$ :

$$
I(p)=I\left(w_{l}, w_{l+1}, \cdots, w_{m}\right)=\sum_{i=l}^{m} I\left(w_{i}\right)
$$

where $I(w)$ is the surprisal of subword $w$ :

$$
I\left(w_{i}\right)=-\log p\left(w_{i} \mid w_{<i}\right)
$$

For the statistical analyses, we first trained a baseline regression model with several predictors that are known to affect reading times. Then, we added surprisal estimated from each LM as a predictor and evaluated the decrease in deviance $(\Delta D(L M))$ as psychometric predictive power: ${ }^{5}$

$$
\Delta D(L M)=D_{B}-D_{L M}
$$

where $D_{B}$ and $D_{L M}$ are deviance of the baseline regression model and the regression model with surprisal, respectively. The details of our regression models are shown in Appendix A.

Perplexity and parsing accuracy: Goodkind and Bicknell (2018) demonstrated that perplexity of LMs and their psychometric predictive power are

\footnotetext{
${ }^{5}$ In the previous literature, surprisals of the previous segments were also added as a predictor to capture spillover effects (Smith and Levy, 2013). In our experiments, we did not add surprisals of the previous segments because preliminary experiments showed that they were not significant for modeling reading times in all the LMs.
} 
highly correlated. In order to investigate whether this correlation can be observed, perplexities of LMs were calculated based on the sentences in BCCWJ-EyeTrack.

In addition, given that RNNGs also serve as a parser, the correlation between parsing accuracy and psychometric predictive power was also investigated. The evaluation metric of parsing accuracy was the labeled bracketing F1. For this purpose, we used the sentences in NPCMJ because the sentences in BCCWJ-EyeTrack are not annotated with tree structures. Parsing accuracies of RNNGs were calculated based on the tree structures at the top of the final beam in word-synchronous beam search.

\section{Results and discussion}

The result of our computational modeling is summarized in Figure 1: psychometric predictive power (the vertical axis) is plotted against perplexity (the horizontal axis). ${ }^{6}$ In this section, we first analyze psychometric predictive power itself, and then discuss its relationships with (i) perplexity, (ii) parsing, and (iii) beam size.

\subsection{Psychometric predictive power}

Figure 1 demonstrates that the hierarchical models (top-down/left-corner RNNGs) achieved higher psychometric predictive power than the sequential model (LSTM) and, among the hierarchical models, left-corner RNNGs achieved higher psychometric predictive power than top-down RNNGs. In order to confirm that these differences are statistically meaningful, we performed nested model comparisons. The result of nested model comparisons is summarized in Table 1, where the best result from each LM was compared. ${ }^{7}$ The significance threshold at $\alpha=0.0056$ was imposed by the Bonferroni correction motivated by 9 tests $(0.05 / 9)$.

First, nested model comparisons revealed that psychometric predictive power was significant for all LMs relative to the baseline regression model. The point here is that surprisals computed by LMs do explain human reading times in Japanese, generalizing the previous results in English.

Second, the hierarchical models (top-down/leftcorner RNNGs) significantly outperformed the sequential model (LSTM), and the sequential model

\footnotetext{
${ }^{6}$ The lower perplexity means the better next-subword prediction accuracy.

${ }^{7}$ Top-down RNNGs achieved the best result with the action beam size 1000, while left-corner RNNGs achieved the best result with the action beam size 400 .
}

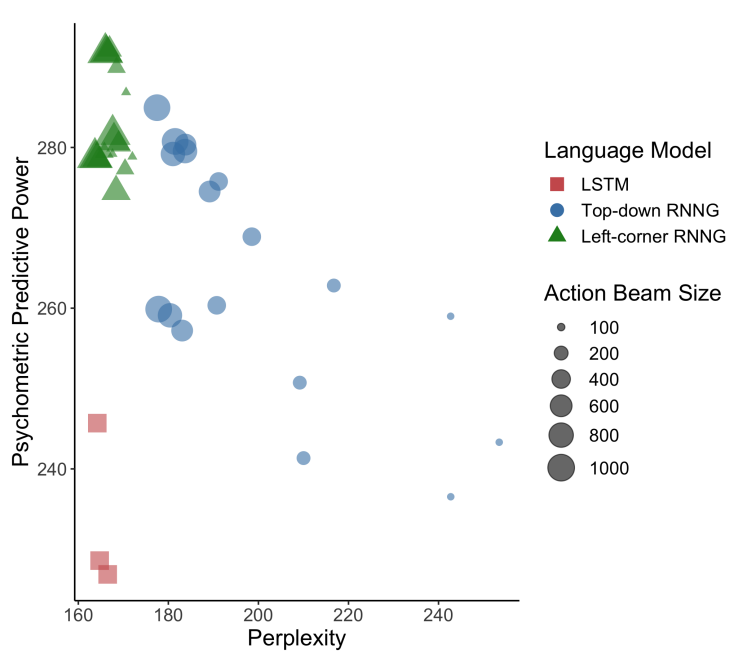

Figure 1: The result of our computational modeling: psychometric predictive power (the vertical axis) is plotted against perplexity (the horizontal axis).

did not account for unique variances that the hierarchical models cannot explain. This result aligns with the previous observation that LMs become more human-like by explicitly modeling hierarchical structures on top of linear strings (Kuncoro et al., 2018; Wilcox et al., 2019; Hale et al., 2018).

Finally, among the hierarchical models, leftcorner RNNGs significantly outperformed topdown RNNGs, and top-down RNNGs did not account for unique variances that left-corner RNNGs cannot explain. This result corroborates Abney and Johnson (1991) from an information-theoretic perspective: the left-corner parsing strategy is more cognitively plausible than the top-down and bottom-up paring strategies.

Here we can conclude from these results that LMs become more human-like by explicitly modeling hierarchical structures and, most importantly, the left-corner parsing strategy was more cognitively plausible than the top-down parsing strategy.

\subsection{Perplexity}

In this subsection, we discuss the relationship between perplexity and psychometric predictive power. First, Figure 1 indicates that, among the hierarchical models, left-corner RNNGs, which achieved higher psychometric predictive power, also achieved lower perplexity than top-down RNNGs. Overall, the correlation between perplexity and psychometric predictive power of the hierarchical models was robust: the lower perplexity RNNGs have, the higher psychometric predictive power they also have. In sharp contrast, the correlation did not hold for the sequential model, where 296 STMs achieved better perplexity, but worse psy- 


\begin{tabular}{lccc}
\hline & $\chi^{2}$ & df & $p$ \\
\hline Baseline < LSTM & 260.26 & 1 & $<\mathbf{0 . 0 0 0 1}$ \\
Baseline < TD & 299.5 & 1 & $<\mathbf{0 . 0 0 0 1}$ \\
Baseline < LC & 306.78 & 1 & $<\mathbf{0 . 0 0 0 1}$ \\
LSTM $<$ TD & 42.183 & 1 & $<\mathbf{0 . 0 0 0 1}$ \\
LSTM $<$ LC & 47.229 & 1 & $<\mathbf{0 . 0 0 0 1}$ \\
TD $<$ LSTM & 2.9406 & 1 & 0.08638 \\
TD $<$ LC & 11.84 & 1 & $<\mathbf{0 . 0 0 1}$ \\
LC $<$ LSTM & 0.708 & 1 & 0.4001 \\
LC $<$ TD & 4.5609 & 1 & 0.03271 \\
\hline
\end{tabular}

Table 1: The result of nested model comparisons with the best result from each LM. TD and LC indicate topdown and left-corner RNNGs, respectively. The significance threshold at $\alpha=0.0056$ was imposed by the Bonferroni correction motivated by 9 tests $(0.05 / 9)$.

chometric predictive power than RNNGs with similar or even worse perplexity, corroborating Goodkind and Bicknell (2018) that LSTM stands out as an outlier of the correlation between perplexity and psychometric predictive power.

Kuribayashi et al. (2021) recently showed that the correlation between perplexity and psychometric predictive power cannot be generalized to Japanese. They proposed that LMs are trained to flatten information density and thus satisfy the Uniform Information Density (UID) assumption (Genzel and Charniak, 2002; Levy, 2005; Jaeger and Levy, 2007), but information density in Japanese turned out not to be empirically uniform and far from the idealized UID assumption. At first, this proposal appears to be inconsistent with our results, but notice that Kuribayashi et al. (2021) only tested sequential models. Here we would like to suggest that, unlike sequential models, hierarchical models can be trained to be human-like, even in languages far from the idealized UID assumption.

\subsection{Parsing}

In this subsection, we discuss the relationship between parsing accuracy and psychometric predictive power, which is summarized in Appendix B, where psychometric predictive power (the vertical axis) is plotted against parsing accuracy (the horizontal axis). Interestingly, just like perplexity, left-corner RNNGs, which achieved higher psychometric predictive power, also achieved higher parsing accuracy than top-down RNNGs. Here again, the correlation between parsing accuracy and psychometric predictive power of the hierarchi- cal models was robust: the higher parsing accuracy RNNGs have, the higher psychometric predictive power they also have.

\subsection{Beam size}

Finally, we discuss the relationship between beam size and psychometric predictive power. The important generalization here is that, although topdown RNNGs improved in psychometric predictive power, perplexity, and parsing accuracy only when the beam size increased, left-corner RNNGs consistently performed well even with a small beam size. We interpret this generalization as demonstrating that left-corner RNNGs may be more human-like than top-down RNNGs in that they can infer the correct tree structure even with a small beam size comparable to humans. In order to reinforce this reasoning, we discuss (i) why left-corner RNNGs can infer the correct tree structure with a small beam size, and (ii) why inference with a small beam size is comparable to humans.

First, we show why left-corner RNNGs can infer the correct tree structure with a small beam size. Consider the following left-branching structures:
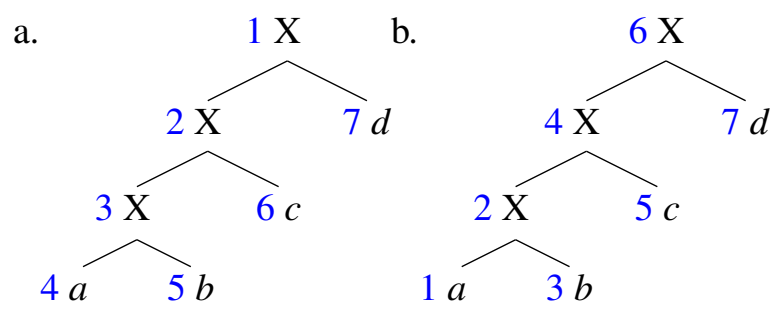

The structures (a) and (b) represent the order in which nodes are computed by the top-down and the left-corner parsing strategies, respectively. In the top-down parsing strategy, all the ancestor nodes of $a$ must be enumerated before processing $a$. Only when the beam size increases, it is possible to assume various depths and nodes, and thus retain the correct tree structure during sentence processing. On the other hand, in the left-corner parsing strategy, where the mother node is enumerated after its leftmost child, it is not necessary to enumerate the ancestor nodes before processing $a$. Thus, the correct tree structure can be inferred with a small beam size via the left-corner parsing strategy.

Second, we show why inference with a small beam size is comparable to humans. In fact, Jurafsky (1996) suggested that full parallel processing and serial processing are not appropriate for human sentence processing, and instead partial parallel processing via beam search that prunes low prob- 
ability structures is cognitively plausible. From this perspective, we may argue that inference with a small beam size is comparable to humans, and left-corner RNNGs, which can infer the correct tree structure with a small beam size, may be more human-like than top-down RNNGs.

As an anonymous reviewer correctlly pointed out, however, given that Jurafsky (1996) proposed beam search that only keeps structures with a probability within a multiple of 3.8 to 5.6 of the probability of the most probable structure, the number of structures within such a relative beam could be extremely large, especially if the probabilities of the structures within the beam are similar. In order to address this point, we computed an empirical beam size comparable to humans. Specifically, we calculated the number of structures with a probability more than $1 / 3.8$ and $1 / 5.6$ of the probability of the most probable structure, among structures within the word beam which corresponds to the beam defined in Jurafsky (1996). The results showed that, even with the largest word beam size of 100 , the average number of structures through derivations within the proposed relative beam turned out to be empirically small: between 3.05 and 4.14 for top-down RNNGs and between 4.08 and 5.68 for left-corner RNNGs. The details of the results are shown in Appendix C. We believe that these results do not affect our argument that inference with a small beam size is comparable to humans.

These discussions taken together, we could still argue that left-corner RNNGs, which can infer the correct tree structure with a small beam size, may be more human-like than top-down RNNGs. In addition, given that larger beam sizes make LMs more computationally expensive, these results also suggest that left-corner RNNGs are more efficient.

\section{Limitations and future work}

Interestingly, Wilcox et al. (2020) demonstrated that top-down RNNGs underperformed LSTMs in predicting human reading times in English, which appears to be contradictory to our results in Japanese. We would like to suggest that this discrepancy can be attributed to the difference in the languages tested in these papers. In fact, Kuribayashi et al. (2021) have shown that several established results in English cannot be straightforwardly generalized to Japanese.

In addition, Wilcox et al. (2020) found that $n$ gram language models outperformed various neural language models, while Merkx and Frank (2021) observed that Transformers (Vaswani et al., 2017) outperformed LSTMs in modeling self-paced reading times and N400 brain activities, but not in predicting eye-tracking reading times.

In order to address these limitations, we plan to conduct detailed comparisons between English (Dundee Corpus, Kennedy et al., 2003) and Japanese (BCCWJ-EyeTrack, Asahara et al., 2016) with RNNGs, incorporating $n$-gram language models and Transformers as baselines in future work.

\section{Conclusion}

In this paper, we investigated whether hierarchical structures make LMs more human-like, and if so, which parsing strategy is most cognitively plausible. Our computational modeling demonstrated that left-corner RNNGs outperformed top-down RNNGs and LSTM, suggesting that hierarchical and left corner architectures are more cognitively plausible than top-down or sequential architectures. Moreover, lower perplexities and higher parsing accuracies of the hierarchical models were strongly correlated with the higher psychometric predictive power, but the correlation did not hold for the sequential model. In addition, left-corner RNNGs may be more human-like than top-down RNNGs in that they can infer the correct tree structure with a small beam size comparable to humans.

\section{Acknowledgements}

We would like to thank three anonymous reviewers for their insightful suggestions. This work was supported by JSPS KAKENHI Grant Number 19H04990 and the National Institute for Japanese Language and Linguistics (NINJAL) Collaborative Research Project "Computational Psycholinguistics of Language Processing with Large Corpora."

\section{References}

Steven P. Abney and Mark Johnson. 1991. Memory requirements and local ambiguities of parsing strategies. Journal of Psycholinguistic Research, 20(3):233-250.

Masayuki Asahara, Kikuo Maekawa, Mizuho Imada, Sachi Kato, and Hikari Konishi. 2014. Archiving and analysing techniques of the ultra-large-scale web-based corpus project of ninjal, japan. Alexandria, 25:129-148.

Masayuki Asahara, Hajime Ono, and Edson T. Miyamoto. 2016. Reading-Time Annotations 
for "Balanced Corpus of Contemporary Written Japanese". In Proceedings of COLING 2016, the 26th International Conference on Computational Linguistics: Technical Papers, pages 684-694, Osaka, Japan. The COLING 2016 Organizing Committee.

Chris Dyer, Adhiguna Kuncoro, Miguel Ballesteros, and Noah A. Smith. 2016. Recurrent Neural Network Grammars. In Proceedings of the 2016 Conference of the North American Chapter of the Association for Computational Linguistics: Human Language Technologies, pages 199-209, San Diego, California. Association for Computational Linguistics.

Victoria Fossum and Roger Levy. 2012. Sequential vs. Hierarchical Syntactic Models of Human Incremental Sentence Processing. In Proceedings of the 3rd Workshop on Cognitive Modeling and Computational Linguistics, pages 61-69, Montréal, Canada. Association for Computational Linguistics.

Stefan Frank and Rens Bod. 2011. Insensitivity of the Human Sentence-Processing System to Hierarchical Structure. Psychological science, 22:829-34.

Stefan L. Frank, Leun J. Otten, Giulia Galli, and Gabriella Vigliocco. 2015. The ERP response to the amount of information conveyed by words in sentences. Brain and Language, 140:1-11.

Dmitriy Genzel and Eugene Charniak. 2002. Entropy rate constancy in text. In Proceedings of the 40th Annual Meeting of the Association for Computational Linguistics, pages 199-206, Philadelphia, Pennsylvania, USA. Association for Computational Linguistics.

Adam Goodkind and Klinton Bicknell. 2018. Predictive power of word surprisal for reading times is a linear function of language model quality. In Proceedings of the 8th Workshop on Cognitive Modeling and Computational Linguistics, pages 10-18, Salt Lake City, Utah. Association for Computational Linguistics.

Kristina Gulordava, Piotr Bojanowski, Edouard Grave, Tal Linzen, and Marco Baroni. 2018. Colorless Green Recurrent Networks Dream Hierarchically. In Proceedings of the 2018 Conference of the North American Chapter of the Association for Computational Linguistics: Human Language Technologies, Volume 1 (Long Papers), pages 1195-1205, New Orleans, Louisiana. Association for Computational Linguistics.

John Hale. 2001. A Probabilistic Earley Parser as a Psycholinguistic Model. In Proceedings of the Second Conference of the North American Chapter of the Association for Computational Linguistics, pages 159-166.

John Hale, Chris Dyer, Adhiguna Kuncoro, and Jonathan Brennan. 2018. Finding syntax in human encephalography with beam search. In Proceedings of the 56th Annual Meeting of the Association for Computational Linguistics (Volume 1: Long Papers), pages 2727-2736, Melbourne, Australia. Association for Computational Linguistics.

T Jaeger and Roger Levy. 2007. Speakers optimize information density through syntactic reduction. In Advances in Neural Information Processing Systems, volume 19, pages 849-856. MIT Press.

Lifeng Jin and William Schuler. 2020. Memorybounded Neural Incremental Parsing for Psycholinguistic Prediction. In Proceedings of the 16th International Conference on Parsing Technologies and the IWPT 2020 Shared Task on Parsing into Enhanced Universal Dependencies, pages 48-61, Online. Association for Computational Linguistics.

Daniel Jurafsky. 1996. A Probabilistic Model of Lexical and Syntactic Access and Disambiguation. Cognitive Science, 20(2):137-194.

Alan Kennedy, Robin Hill, and Joël Pynte. 2003. The dundee corpus. In Proceedings of the 12th European conference on eye movement.

Taku Kudo and John Richardson. 2018. SentencePiece: A simple and language independent subword tokenizer and detokenizer for Neural Text Processing. In Proceedings of the 2018 Conference on Empirical Methods in Natural Language Processing: System Demonstrations, pages 66-71, Brussels, Belgium. Association for Computational Linguistics.

Adhiguna Kuncoro, Miguel Ballesteros, Lingpeng Kong, Chris Dyer, Graham Neubig, and Noah A. Smith. 2017. What Do Recurrent Neural Network Grammars Learn About Syntax? In Proceedings of the 15th Conference of the European Chapter of the Association for Computational Linguistics: Volume 1, Long Papers, pages 1249-1258, Valencia, Spain. Association for Computational Linguistics.

Adhiguna Kuncoro, Chris Dyer, John Hale, Dani Yogatama, Stephen Clark, and Phil Blunsom. 2018. LSTMs Can Learn Syntax-Sensitive Dependencies Well, But Modeling Structure Makes Them Better. In Proceedings of the 56th Annual Meeting of the Association for Computational Linguistics (Volume 1: Long Papers), pages 1426-1436, Melbourne, Australia. Association for Computational Linguistics.

Tatsuki Kuribayashi, Yohei Oseki, Takumi Ito, Ryo Yoshida, Masayuki Asahara, and Kentaro Inui. 2021. Lower perplexity is not always human-like. In Proceedings of the 59th Annual Meeting of the Association for Computational Linguistics and the 11th International Joint Conference on Natural Language Processing (Volume 1: Long Papers), pages 52035217, Online. Association for Computational Linguistics.

Roger Levy. 2005. Probabilistic models of word order and syntactic discontinuity. stanford university. 
Roger Levy. 2008. Expectation-based syntactic comprehension. Cognition, 106(3):1126-1177.

Christopher Manning and Hinrich Schutze. 1999. Foundations of Statistical Natural Language Processing. MIT Press.

Danny Merkx and Stefan L. Frank. 2021. Human sentence processing: Recurrence or attention? In Proceedings of the Workshop on Cognitive Modeling and Computational Linguistics, pages 12-22, Online. Association for Computational Linguistics.

Hiroshi Noji and Yohei Oseki. 2021. Effective batching for recurrent neural network grammars. In Findings of the Association for Computational Linguistics: ACL-IJCNLP 2021, pages 4340-4352, Online. Association for Computational Linguistics.

Philip Resnik. 1992. Left-corner parsing and psychological plausibility. In Proceedings of the 14th Conference on Computational Linguistics - Volume 1, COLING '92, pages 191-197, USA. Association for Computational Linguistics.

Rico Sennrich, Barry Haddow, and Alexandra Birch 2016. Neural Machine Translation of Rare Words with Subword Units. In Proceedings of the 54th Annual Meeting of the Association for Computational Linguistics (Volume 1: Long Papers), pages 17151725, Berlin, Germany. Association for Computational Linguistics.

Nathaniel J. Smith and Roger Levy. 2013. The effect of word predictability on reading time is logarithmic. Cognition, 128(3):302-319.

Mitchell Stern, Daniel Fried, and Dan Klein. 2017. Effective Inference for Generative Neural Parsing. In Proceedings of the 2017 Conference on Empirical Methods in Natural Language Processing, pages 1695-1700, Copenhagen, Denmark. Association for Computational Linguistics.

Marten van Schijndel and Tal Linzen. 2018. A neural model of adaptation in reading. In Proceedings of the 2018 Conference on Empirical Methods in Natural Language Processing, pages 4704-4710, Brussels, Belgium. Association for Computational Linguistics.

Ashish Vaswani, Noam Shazeer, Niki Parmar, Jakob Uszkoreit, Llion Jones, Aidan N. Gomez, Łukasz Kaiser, and Illia Polosukhin. 2017. Attention Is All You Need. In Proceedings of NIPS, pages 59986008.

Ethan Wilcox, Jon Gauthier, Jennifer Hu, Peng Qian, and Roger Levy. 2020. On the Predictive Power of Neural Language Models for Human Real-Time Comprehension Behavior. In Proceedings of the 42nd Annual Meeting of the Cognitive Science Society, pages 1707-1713.
Ethan Wilcox, Peng Qian, Richard Futrell, Miguel Ballesteros, and Roger Levy. 2019. Structural supervision improves learning of non-local grammatical dependencies. In Proceedings of the 2019 Conference of the North American Chapter of the Association for Computational Linguistics: Human Language Technologies, Volume 1 (Long and Short Papers), pages 3302-3312, Minneapolis, Minnesota. Association for Computational Linguistics. 


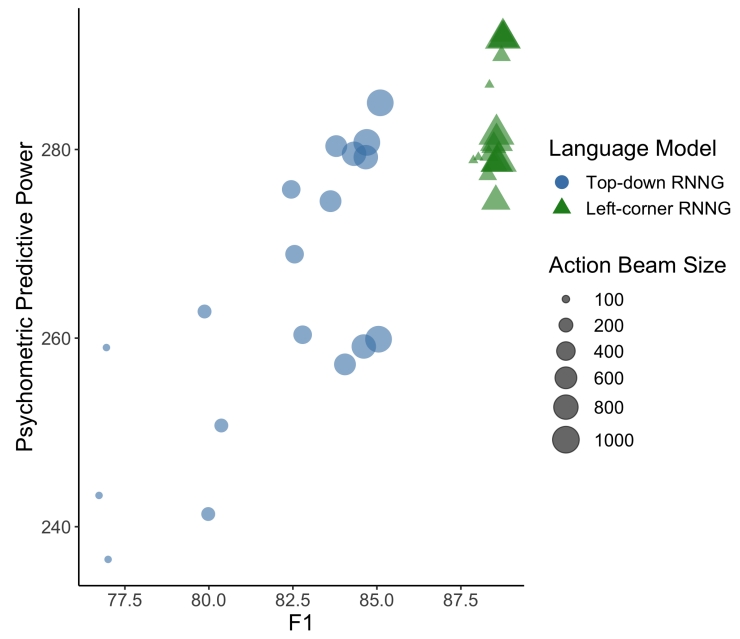

Figure 2: The relationship between parsing accuracy and psychometric predictive power: psychometric predictive power (the vertical axis) is plotted against parsing accuracy (the horizontal axis).

\section{A Details of our regression model}

The logarithmic reading time $(\log (\mathrm{RT}))$ was modeled using the following linear mixed-effects model as a baseline regression model:

$$
\begin{aligned}
\log (\mathrm{RT}) & \sim \text { length }+ \text { prev_length } \\
& + \text { freq }+ \text { prev_freq }+ \text { is_first } \\
& + \text { is_last }+ \text { is_second_last } \\
& + \text { screenN }+ \text { lineN }+ \text { segment } \\
& +(\text { l|article })+(1 \mid \text { subj }) .
\end{aligned}
$$

Table 2 shows descriptions for the factors used in our experiments. We contained predictors and random intercepts that were used in Asahara et al. (2016), and added a predictor related to frequency following the previous literature (e.g., Frank and Bod, 2011; Fossum and Levy, 2012). Frequencies were estimated using the larger National Institute for Japanese Language and Linguistics Web Japanese Corpus (NWJC, Asahara et al., 2014). To capture spillover effects, the length and frequency of the previous segments were also added as a predictor (Smith and Levy, 2013). All numeric factors were centered, and the predictors that were not significant $(p>0.05)$ for modeling reading times were excluded. We removed 27 data points that were beyond three standard deviations. This left 12,087 data points as final statistical analysis targets.

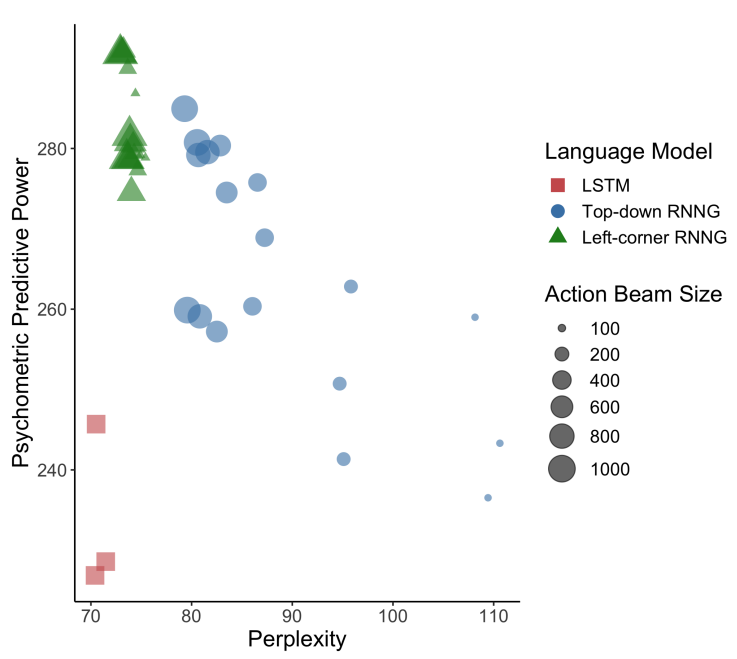

Figure 3: The relationship between perplexity on the NPCMJ test set and psychometric predictive power: psychometric predictive power (the vertical axis) is plotted against perplexity on the NPCMJ test set (the horizontal axis).

\section{B Relationship between parsing accuracy and psychometric predictive power}

The relationship between parsing accuracy and psychometric predictive power is summarized in Figure 2: psychometric predictive power is plotted against parsing accuracy (F1). Just like perplexity, left-corner RNNGs, which achieved higher psychometric predictive power, also achieved the higher parsing accuracy than top-down RNNGs. Overall, the correlation between parsing accuracy and psychometric predictive power of the hierarchical models was robust: the higher parsing accuracy RNNGs have, the higher psychometric predictive power they also have.

\section{Empirical beam size in Jurafsky (1996)}

Table 3 shows the average number of structures through derivations within the beam proposed in Jurafsky (1996). Specifically, we calculated the number of structures with a probability more than $1 / 3.8$ and $1 / 5.6$ of the probability of the most probable structure, among structures within the word beam which corresponds to the beam defined in Jurafsky (1996). The average number of structures within the proposed relative beam turned out to be empirically small. 


\section{Hyparameters and available codes}

Table 4 shows the hyperparameters of LMs. The code of LSTM we employed (Gulordava et al., 2018) is available at https: //github.com/facebookresearch/ colorlessgreenRNNs. Surprisals of LSTM were calculated with the code implemented by van Schijndel and Linzen (2018), which is available at https://github.com/vansky/ neural-complexity. The code of RNNGs we employed (Noji and Oseki, 2021) is also available at https://github.com/aistairc/ rnng-pytorch. We used a byte-pair encoding implemented in sentencepiece (Kudo and Richardson, 2018), which is available at https: //github.com/google/sentencepiece. We set character coverage to 0.9995 , and vocabulary size to 8,000 .

\section{E Dataset split ratio}

Sentences in the training data, NPCMJ, are from 14 sources. We used $90 \%$ of sentences in each source as a training set, and $5 \%$ of sentences as a validation set. The remaining 5\% were used as a test set to calculate paring accuracies of RNNGs in Section 4 and perplexities of LMs in Appendix F.

\section{F Relationship between perplexity on the NPCMJ test set and psychometric predictive power}

We additionally investigated the relationship between perplexity calculated based on the sentences in the NPCMJ test set and psychometric predictive power. The result is shown in Figure 3: psychometric predictive power is plotted against perplexity on the NPCMJ test set. Although the perplexities of all LMs were overall lower, there was no substantial difference with the result shown in Figure 1. The difference in the corpus domain may cause the overall difference in perplexity. 


\begin{tabular}{|c|c|c|}
\hline Name & Type & Description \\
\hline length & int & Number of characters in the segment \\
\hline prev_length & int & Number of characters in the previous segment \\
\hline freq & num & $\begin{array}{l}\text { Logarithm of the geometric mean of the word frequencies in the } \\
\text { segment }\end{array}$ \\
\hline prev_freq & num & $\begin{array}{l}\text { Logarithm of the geometric mean of the word frequencies in the } \\
\text { previous segment }\end{array}$ \\
\hline is_first & factor & Whether the segment is the first on a line \\
\hline is_last & factor & Whether the segment is the last on a line \\
\hline is_second_last & factor & Whether the segment is the second to last on a line \\
\hline screenN & int & Screen display order \\
\hline lineN & int & Line display order \\
\hline segmentN & int & Segment display order \\
\hline article & factor & Article ID \\
\hline subj & factor & Participant ID \\
\hline
\end{tabular}

Table 2: Descriptions for the factors used in our experiments.

\begin{tabular}{llcccccc}
\hline & & \multicolumn{5}{c}{ Word beam size } \\
\cline { 3 - 7 } Model & Threshold & 10 & 20 & 40 & 60 & 80 & 100 \\
\hline TD & $1 / 3.8$ & $1.67( \pm 0.0)$ & $2.14( \pm 0.0)$ & $2.61( \pm 0.0)$ & $2.81( \pm 0.1)$ & $2.95( \pm 0.1)$ & $3.05( \pm 0.1)$ \\
& $1 / 5.6$ & $1.95( \pm 0.0)$ & $2.62( \pm 0.0)$ & $3.35( \pm 0.1)$ & $3.69( \pm 0.1)$ & $3.95( \pm 0.1)$ & $4.14( \pm 0.1)$ \\
\hline LC & $1 / 3.8$ & $2.58( \pm 0.1)$ & $3.08( \pm 0.1)$ & $3.53( \pm 0.2)$ & $3.83( \pm 0.1)$ & $4.01( \pm 0.1)$ & $4.08( \pm 0.2)$ \\
& $1 / 5.6$ & $3.14( \pm 0.1)$ & $3.93( \pm 0.2)$ & $4.73( \pm 0.2)$ & $5.21( \pm 0.2)$ & $5.52( \pm 0.2)$ & $5.67( \pm 0.3)$ \\
\hline
\end{tabular}

Table 3: The average number of structures with a probability more than $1 / 3.8$ and $1 / 5.6$ of the probability of the most probable structure, among structures within the word beam which corresponds to the beam defined in Jurafsky (1996). TD and LC indicate top-down and left-corner RNNGs, respectively. Average scores with standard deviations across different random seeds are reported.

\begin{tabular}{llc}
\hline LSTM & optimizer & SGD \\
& learning rate & 20 \\
& dropout & 0.2 \\
& batch size & 64 \\
\hline Top-down RNNGs & optimizer & adam \\
& learning rate & 0.001 \\
& dropout & 0.3 \\
& batch size & 64 \\
\hline Left-corner RNNGs & optimizer & adam \\
& learning rate & 0.001 \\
& dropout & 0.3 \\
& batch size & 64 \\
\hline
\end{tabular}

Table 4: Hyperparameters of LMs. 